immunosuppressive cytotoxic chemotherapy is likely to improve the outcome for these patients. Further clinical and basic studies should demonstrate the role of these agents in diseases such as post transplant and endemic Burkitt’s lymphomas.

\title{
Epidemiology of Virus-Associated Cancers in Brazil Rocío Hassan
}

Epidemiological evidences point to an increasing causative role of viruses in the multifactorial multistep etiology of human malignancies. The interactions of viruses with certain environmental and genetic factors may account for the geographic variability of incidences and clinic-biological characteristics of particular virus-associated cancers. Estimates about the preventable risks of cancer in the U.S. showed that approximately $10 \%$ of human cancers were causatively associated with viruses. In developing countries, interaction with other etiologic and risk factors besides the size of the "at risk" populations ( $80 \%$ of human population), act synergically to increase the magnitude of this public health problem. In most of the cases, an accurate characterization of the problem is hampered by the lack of interdisciplinary studies in those areas. In Brazil, the main associations are HPV and cervical cancer, HTLVI/II and T cell leukemia and EBV -associated neoplasms, all of them transversally influenced by the HIV epidemics. Due to the continental dimensions of the country, investigations on prevalence, viral infection patterns and association with other environmental, genetic and susceptibility factors are needed, on a regional basis, for identifying risk areas and virus-host specific interactions involved in viral associated carcinogenesis, aiming to the design of prevention and therapeutic strategies.

We studied a group of 62 children with Burkitt's lymphoma (BL) at the National Institute of Cancer, (INCa), Rio de Janeiro. Association with Epstein-Barr Virus (EBV) was found in 60\% of the cases (86\% of type 1), thus confirming an intermediate prevalence in the Southeast region of the country, compared to the Northeast region, where it can reach 87\%. The four children diagnosed with AIDS-BL were EBV-positive. Children with EBV+ $B L$ were younger than those with $\mathrm{EBV}-\mathrm{BL}(\mathrm{p}=0.002)$. Aiming to identify susceptibility factors, we studied the IL10 promoter polymorphisms SNP-1082(G/A) and STR IL10.G, as well as TNF $\alpha$ SNP-308(G/A). We found an excess of the highly expressing allele SNP-1082 (G) in EBV-negative patients (OR 2.82; CI 95\% 1.22-6.51; $\mathrm{p}=0.013$ ) pointing to a stimulatory, pro-oncogenic role of the IL-10 in this group. In 15 BL patients we investigated the expression of cellular genes by RT-PCR and found that cyclin D2 was expressed in $53 \%$ of the cases, associated to the presence of EBV ( $\mathrm{p}=0.041)$. BCL2 was expressed in 4 of 13 cases (31\%). Expression of activation induced cytidine deaminase (AID) gene, which is involved in physiological maturation and remodelling of immunoglobulin genes was quantified by Real-time PCR. We found a higher than expected expression of AID in the EBV-positive cases, not associated to the presence or degree of somatic hypermutation of IGH locus, which may be reflecting interactions of viral and cellular regulatory pathways. In sum, we presented results of current research in our laboratory which is helping us to understand the complex interactions between virus and host in the pathogenesis of Burkitt‘s lymphoma in this region.

\section{Epidemiology of AIDS-Related Malignancies in Bahia Eduardo M Netto, Estela Luz, Carlos Brites}

Brazil reported up to June 2004 more than 362,000 individuals with AIDS (and 160,000 deaths) most of all with full blow disease, with a cumulative incidence rate of $18.4 / 100,000$ habitants. The number of cases is rather 